\title{
Impact of the COVID-19 pandemic on mental health of pregnant women with diabetes mellitus and hypertension
}

\author{
Roberta de Pádua Borges ${ }^{1 *}$ (1), Angela de Azevedo Jacob Reichelt ${ }^{2}$ (D), Ariane de Brito ${ }^{3}$ (D), \\ Gabriela Oliveira Gonçalves Molino ${ }^{4}$, Beatriz D. Schaan ${ }^{1,2}$ (i)
}

\section{SUMMARY}

OBJECTIVE: Psychological effects of the coronavirus disease 2019 (COVID-19) pandemic on pregnant women with diabetes and hypertension are not yet studied. Besides the pregnancy, these women have additional risk factors for severe acute respiratory syndrome due to COVID-19 and are considered a particularly vulnerable, unique population. We aimed to assess their mental health during this pandemic.

METHODS: This is a cross-sectional study carried out at a Brazilian tertiary hospital. Women with pregnancies complicated by hypertension and/or diabetes were evaluated. The primary outcome was anxiety, and depressive symptoms evaluated with the State-Trait Anxiety Inventory and Patient Health Questionnaire. Perception of changing habits during quarantine was evaluated as a secondary outcome. RESULTS: Seventy-nine patients were included. The prevalence of State-Trait Anxiety Inventory $\geq 40$ was $79.7 \%$ and that of Patient Health Questionnaire $\geq 10$ was $59.2 \%$. Lower social support was correlated with higher scores on both scales. Time spent with electronic devices was perceived as greater by $62 \%$ of the women.

CONCLUSIONS: Pregnant women with diabetes and hypertension presented high levels of anxiety and depressive symptoms during the COVID-19 pandemic. Considering that these symptoms can affect both the mother's and offspring's health, it is necessary to implement tools to improve their mental health.

KEYWORDS: COVID-19. Pregnancy. Mental health. Anxiety. Diabetes mellitus. Hypertension.

\section{INTRODUCTION}

Anxiety disorders in pregnancy are frequent. Prolonged anxiety during pregnancy has been associated with premature birth, fetal growth restriction, and children's behavioral problems ${ }^{1}$, as well as maternal postpartum depression ${ }^{2}$.

In December 2019, a case series of respiratory syndromes caused by a new coronavirus, a disease later named COVID-19, was described in China. By May 2021, more than 150 million cases had been confirmed in the world. Since then, Brazil has recorded the second highest number of deaths in the world. To fight the pandemic, measures of hygiene care, social isolation, and use of face masks were established. Social and financial repercussion of these resolutions contributed to the pandemic negative impact on mental health, even to pregnant women. In this specific group, there was also an increase in concerns such as potential exposure to the virus during appointments with health care professionals, especially potential fetus complications in case of infections. The severity of the psychological impact of COVID-19 on pregnant women was shown in an Italian

\footnotetext{
${ }^{1}$ Universidade Federal do Rio Grande do Sul, Graduate program in Medical Science: Endocrinology - Porto Alegre (RS), Brazil.

${ }^{2}$ Hospital de Clínicas de Porto Alegre, Endocrinology division - Porto Alegre (RS), Brazil.

${ }^{3}$ Universidade Federal do Rio Grande do Sul, Graduate program in Psychology - Porto Alegre (RS), Brazil.

${ }^{4}$ Universidade Federal de Ciências da Saúde de Porto Alegre, School of Medicine - Porto Alegre (RS), Brazil.

*Corresponding author: rpborges@hcpa.edu.br

Conflicts of interest: the authors declare there are no conflicts of interest. Funding: none.

Received on July 19, 2021. Accepted on July 20, 2021.
} 
study that evaluated 100 patients, in which about two-thirds presented exacerbation of anxiety symptoms ${ }^{3}$.

Data suggest that people with hypertension and diabetes are more likely to develop severe clinical presentations of COVID-194. Likewise, physiological changes during pregnancy can make pregnant women more vulnerable to serious infections $s^{5}$. Thus, the emotional burden of the current pandemic may be greater in pregnant women with diabetes and hypertension. In addition, treatment of these comorbidities is hampered by the need for social isolation, which can lead to changes in eating habits and an increase in sedentary lifestyle.

Considering that pregnant women with diabetes and hypertension may be particularly vulnerable to the psychological impact caused by the COVID-19 pandemic, and that there are no studies focusing on this specific population. This study aimed to evaluate the impact of adversities caused by the COVID-19 pandemic on mental health of this unique group.

\section{METHODS}

This is a cross-sectional study carried out at a public university hospital in Southern Brazil. We included pregnant women with one or more of the following diagnoses: gestational hypertension, chronic hypertension prior to pregnancy, pregestational type 1 or type 2 diabetes, and gestational diabetes, regardless of gestational age. Exclusion criteria were preeclampsia, eclampsia, or ketoacidosis, since these are serious acute complications that could be confounding factors.

Data collection was carried out by phone calls from July to October 2020. Two trained interviewers were responsible for the calls that lasted about $20 \mathrm{~min}$. Anxiety symptoms were assessed by the State-Trait Anxiety Inventory, in its short version STAI-6. This questionnaire consists of two separate subscales, one measuring trait, and the other state anxiety, STAI-T and STAI-S, respectively. Each subscale consists of six items scored on a 4-point Likert scale. The scores obtained vary from $20-80$, and an abnormal STAI value was considered with results $\geq 40^{6}$.

The Patient Health Questionnaire-9 (PHQ-9), which consists of nine questions that assess the presence of symptoms of major depressive disorder, was also applied. The frequency of each symptom in the previous two weeks is assessed on a four-point Likert scale, ranging from 0 (never) to 3 (almost every day), with higher scores indicating greater severity of depressive symptoms (range 0-27). The recommended cutoff point for being at risk for depressive disorder is $10^{7}$.

To evaluate the perceived social support, the Medical Outcomes Study Social Support Survey (MOS-SSS) was used. It assesses five dimensions of social support: positive social interaction, material, affective, emotional, and informational support.
The STAI, PHQ-9, and MOS-SSS scales were validated for the Brazilian population and are widely used in pregnant women ${ }^{6}$.

Issues related to the changes perceived after the beginning of the confinement period were also addressed: time spent in physical activity, exposure to electronic devices, dietary pattern, and glycemic and/or blood pressure control. Sociodemographic and clinical data were also collected through electronic medical records.

The primary outcomes were the presence of positive screening for anxiety and depression. The secondary outcome was the perception of changing habits. Women with evidence of psychological distress received a brief intervention with weekly telephone follow-up assessments and were referred to specific health services.

The Shapiro-Wilk test was used to assess the variables normality. Data are represented as frequency and percentage for categorical variables, means or medians and standard deviation, and interquartile ranges or minimum and maximum for continuous variables. Kruskal-Wallis, Pearson, and Mann-Whitney $\mathrm{U}$ tests were performed. The scales' internal consistencies were analyzed through the Cronbach's alpha coefficient.

Data analysis was performed using the SPSS software (version 21.0, SPSS Inc., Chicago, IL, USA). We used a significance level of $\mathrm{p}<0.05$ for all statistical tests. The recommendations of the STrengthening the Reporting of OBservational studies in Epidemiology were followed for the development of this manuscript.

\section{RESULTS}

Among the 87 eligible patients, three did not answer the contact attempts, and five had no valid phone numbers on the institution's records. Thus, 79 pregnant women were included.

The median age was 32 years (minimum 16 and maximum 46). The median body mass index (BMI) was 31.6 (minimum 18.8 and maximum 48.7). The diagnosis of gestational diabetes was present in $63.3 \%$, previous type 2 diabetes in $17.7 \%$, and type 1 diabetes in $5.1 \%$ of the patients. Prepregnancy hypertension was present in $31.6 \%$ of patients. Regarding the presence of associated mental disorders, $58.2 \%$ had a previous or current psychiatric illness. The other demographic and clinical characteristics are summarized in Table 1.

Data from the PHQ-9 and STAI-S questionnaires are described in Table 2. PHQ- $99^{3} 10$ was present in $59.2 \%$ of patients, and STAI-S 340 in $79.7 \%$. Time spent with electronic devices was perceived as greater during the pandemic by $62 \%$ of the patients, with a median exposure time of $6 \mathrm{~h}$ (minimum $1 \mathrm{~h}$, maximum $18 \mathrm{~h}$ ). Regarding physical activity, $64.6 \%$ of the women reported having reduced its practice and only $3.8 \%$ 
Table 1. Demographic and clinical characteristics of the population.

\begin{tabular}{|c|c|}
\hline & $n=79$ \\
\hline Age (years) & $32(16-46)$ \\
\hline \multirow{3}{*}{ Self-declared color } & White: 46 (58.2) \\
\hline & Black: 18 (22.8) \\
\hline & Brown: 15 (19) \\
\hline \multirow{4}{*}{ Religion } & Catholic: 32 (40.5) \\
\hline & Evangelical: 16 (20.3) \\
\hline & Others: 16 (20.3) \\
\hline & No religion: 15 (19) \\
\hline \multirow{5}{*}{ Education level } & $\begin{array}{l}\text { Illiterate/incomplete elementary } \\
\text { school: } 6 \text { (7.6) }\end{array}$ \\
\hline & $\begin{array}{c}\text { Complete elementary school: } 11 \\
(13.9)\end{array}$ \\
\hline & Incomplete high school: 16 (20.3) \\
\hline & Complete high school: 34 (43) \\
\hline & $\begin{array}{l}\text { Incomplete/complete higher } \\
\text { education: } 12(15.2)\end{array}$ \\
\hline Unemployment & $45(57)$ \\
\hline $\begin{array}{l}\text { Body mass index } \\
\left(\mathrm{kg} / \mathrm{m}^{2}\right) \mathrm{n}=76\end{array}$ & $31.6(18.8-48.7)$ \\
\hline $\begin{array}{l}\text { Gestational age } \\
\text { (weeks)* }\end{array}$ & $27.7(7.5)$ \\
\hline $\begin{array}{l}\text { Unintended } \\
\text { pregnancy }\end{array}$ & $28(35.4)$ \\
\hline \multirow{4}{*}{ Diabetes mellitus } & Yes: $68(86.1)$ \\
\hline & Gestational diabetes: 50 (63.3) \\
\hline & Type 1 diabetes: 4 (5.1) \\
\hline & Type 2 diabetes: 14 (17.7) \\
\hline Insulin use & $24(30.4)$ \\
\hline Metformin use & $19(24.1)$ \\
\hline \multirow{3}{*}{$\begin{array}{l}\text { Hypertensive } \\
\text { syndromes }\end{array}$} & Yes: $30(38)$ \\
\hline & Gestational hypertension: 5 (6.3) \\
\hline & $\begin{array}{c}\text { Pregestational hypertension: } 25 \\
\text { (31.6) }\end{array}$ \\
\hline $\begin{array}{l}\text { Hypertension and } \\
\text { diabetes association }\end{array}$ & $19(24.1)$ \\
\hline \multirow{3}{*}{ Psychiatric disorder } & Yes: 46 (58.2) \\
\hline & Previous: 22 (27.8) \\
\hline & Current: 24 (30.4) \\
\hline $\begin{array}{l}\text { History of previous } \\
\text { abortion }\end{array}$ & $23(29.1)$ \\
\hline \multirow{3}{*}{$\begin{array}{l}\text { Previous coronavirus } \\
\text { infection }\end{array}$} & No: 69 (87.3) \\
\hline & Suspected: 8 (10.1) \\
\hline & Confirmed: 2 (2.5) \\
\hline
\end{tabular}

Data reported as median (minimum and maximum value) or $n$ (\%). *Evaluated at the time of the questionnaire's application.
Table 2. Results from the STAI-S and PHQ-9 questionnaires.

\begin{tabular}{l|c}
\hline Questionnaire & $\mathrm{n}=79$ \\
STAI-S & $50(40-60)$ \\
\hline STAI-S $\geq 40$ & $63(79.7)$ \\
\hline PHQ-9 & $11(7-16)$ \\
\hline PHQ-9 $\geq 10$ & $47(59.5)$ \\
\hline
\end{tabular}

STAI-S (State-Trait Anxiety Inventory, state subscale), with higher scores indicating greater severity of anxiety symptoms (range 20-80). PHQ-9 (Patient Health Questionnaire-9), with higher scores indicating greater severity of depressive symptoms (range $0-27$ ). Data reported as median (25th and 75 th percentiles) or $n$ (\%).

reported having increased it. Eating habits were described as better by $69.6 \%$ of the patients, and they attributed the concern with the fetus as a propellant for this greater care. Despite this, $53.2 \%$ of pregnant women reported the impression that blood pressure and/or glycemic control got worse during the pandemic (Figure 1).

The score distribution of the STAI-S and the PHQ-9 was similar among women with different comorbidities, age, color, education status, religion, BMI, the number of previous pregnancies, and gestational age, but differed from women with desired compared with those with unintended pregnancies. The median scores of STAI-S and PHQ-9 among women with unintended pregnancies were 58.3 (minimum 23.3 and maximum 80) and 13 (4-27), compared to 46.7 (20-80) and 9 (1-27) among women with desired pregnancies $(\mathrm{p}=0.036$ and $\mathrm{p}=0.004$, respectively). In addition, lower social support was correlated with higher scores on the STAI-S6 scale (Pearson's correlation $-0.273, \mathrm{p}=0.015)$ and with higher scores on the PHQ-9 scale (Pearson's correlation $-0.519, \mathrm{p}<0.001$ ).

The scales' internal consistencies were analyzed through the Cronbach's alpha coefficient. It displayed satisfactory reliability with values of $0.949,0.779$, and 0.775 for the MOS-SSS, PHQ-9, and STAI-S questionnaires, respectively.

\section{DISCUSSION}

The prevalence of symptoms of depression and anxiety in women with high-risk pregnancies complicated by diabetes and hypertension was high. These symptoms were even more frequent in women with less social support and in those with unintended pregnancies. These results are aligned with what has been described in the literature ${ }^{8,9}$. A study comparing two cohorts, with a total of 1,754 pregnant women, one before the pandemic and other during social isolation, found that women evaluated during the pandemic had higher rates of depressive symptoms and anxiety? 


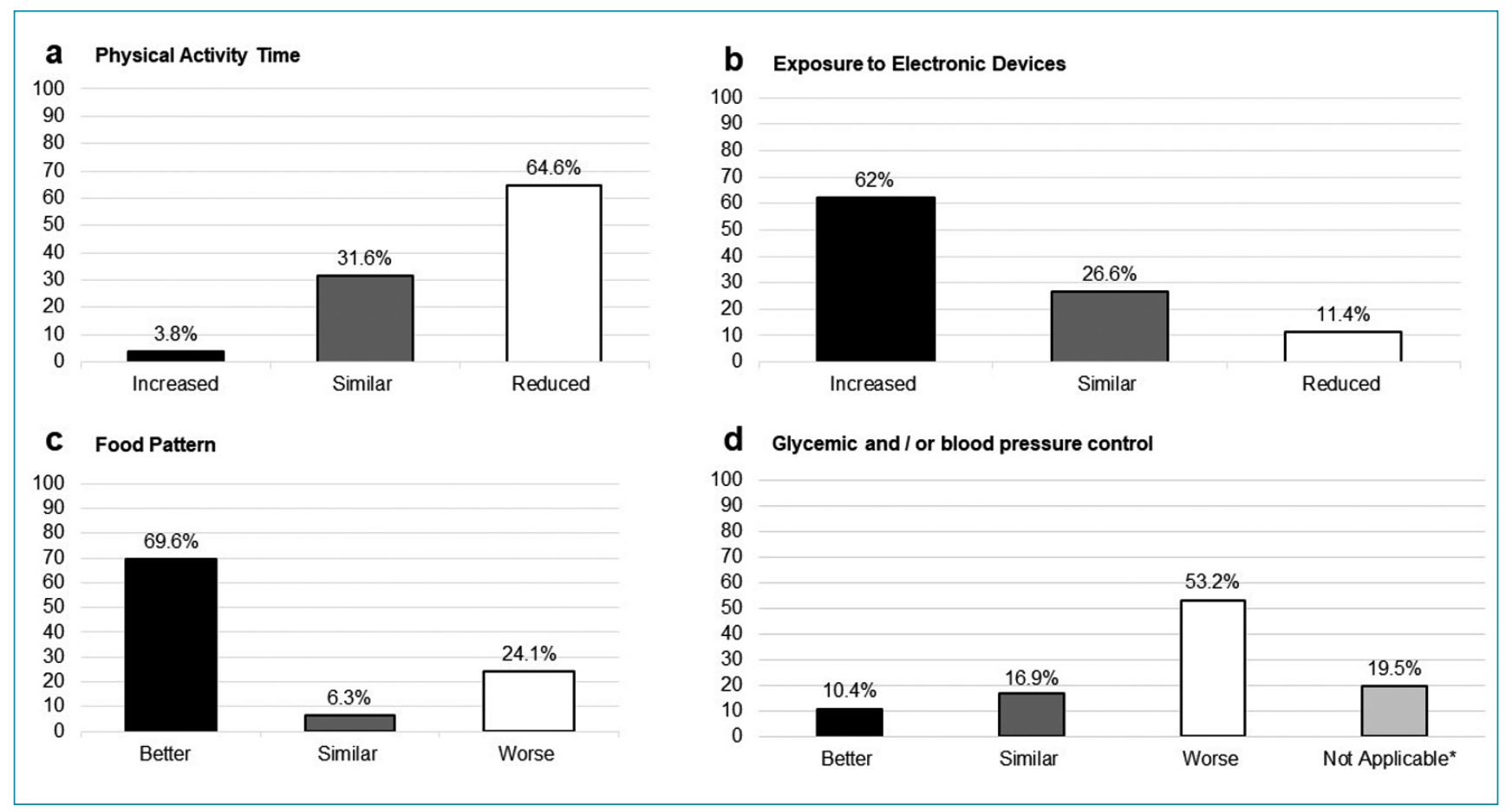

Figure 1. Perceived changes after the COVID-19 quarantine period in relation to the patient's usual routine. Different routine elements were addressed: (A) Changes in the time spent on physical activity; (B) changes in the duration of electronic devices exposure; (C) dietary pattern; and (D) alteration on the glycemic and/or blood pressure control. *Pregnant women with comorbidities diagnosed during pregnancy or who do not control them at home.

During the COVID-19 pandemic, a study by Yassa et al. ${ }^{10}$ found a mean STAI-S score of 41.96 (SD 9.15) in women with healthy pregnancies, with $62.6 \%$ of them presenting scores 340 . In another study, women with high-risk pregnancy indicators demonstrated high levels of anxiety during the ongoing pandemic, with an average STAI-S of 52.55, close to that found in our sample ${ }^{11}$.

Like our results, Sinaci et al. ${ }^{11}$ also found higher levels of anxiety during the pandemic in those women with unplanned pregnancies. Furthermore, our finding of less psychological impact in those with higher perceived levels of social support, corroborates findings of several studies, in which having social support can reduce the risk of depression during pregnancy and postpartum ${ }^{12}$.

Increasing evidence supports the idea that exercise during pregnancy is beneficial for fetal and maternal health. Among benefits for the fetus are adequacy of birth weight ${ }^{13}$, cardiovascular benefits, and possible reduction in the risk of chronic diseases in adulthood ${ }^{14}$. As for the mother, it is suggested a reduction in gestational weight gain ${ }^{15}$, decreased risk of developing preeclampsia ${ }^{13}$, and decreased cesarean section rates, among oth$\mathrm{ers}^{16}$. In our study, $64.6 \%$ of women reported that they have reduced the practice of physical activity. A study evaluating the lifestyle of Spanish pregnant women during the pandemic indicated, like our findings, a significant decrease in their practice of physical activity, but, in contrast, there were no changes in their dietary pattern ${ }^{17}$. In relation to sedentary behavior, the same study found a median of $8 \mathrm{~h}$ of time spent sitting per day, whereas our study showed a median daily screen time of $6 \mathrm{~h}$. Excessive sitting time seems to be harmful, regardless of meeting the recommendations for physical activity ${ }^{18}$. In addition, sedentary maternal behavior during pregnancy seems to negatively affect the outcomes for mother and child ${ }^{19}$.

Our study has strengths. To our knowledge, this is the first study that evaluated this specific population in Brazil, a medium income country with chronically deficient financing of its public health system. In addition to the challenges inherent to the health issues of the pandemic, the country went through a serious political crisis, with exchanges of health ministers, misinformation, and leadership vacuum, a process that reduced confidence in science and government and worsened insecurity among Brazilians ${ }^{20,21}$.

The questionnaires application was carried out by telephone contact to avoid selection bias. According to data from the Brazilian Institute of Geography and Statistics, in 2018, the percentage of households in which there was no Internet 
use exceeded $20 \%$, while the lack of access to a telephone did not exceed 3\% in the South region ${ }^{22}$. Data from the World Health Organization suggest that in 2019, the proportion of women using the Internet globally was $48 \%{ }^{23}$. Despite this, most studies that assessed mental health during the pandemic used online surveys, so a large part of the population may have not been represented.

The use of questionnaires through telephone and virtual interviews has been widely used and is approved by Ethics Committees as long as privacy is guaranteed. Telephone-administered PHQ-9 has already been validated ${ }^{24}$. Studies that directly compare telephone and face-to-face interviewing tend to conclude that telephone administration produces data that are at least comparable in quality to those attained by the face-to-face method, with the advantage to increase accessibility ${ }^{25}$. Further development and evaluation are needed to support the large-scale use of online and telephone-administered assessment programs and questionaries for mental disorders, especially for moments when social distancing is imperative.

Our study differs from others that evaluated high-risk pregnancies since they also included women with unfavorable obstetric history not related to metabolic disorders, such as threat of premature labor, placenta previa, among others ${ }^{11}$. These complications do not appear to pose a greater risk of serious COVID-19 infection.

The small sample size is a limitation of the study. However, we included the entire population that met the inclusion criteria being assisted in our hospital during the established period, with the allowance of $10 \%$ for missing. Other limitations that may limit generalization of findings are the uncontrolled cross-sectional design and having been carried out in a single center. In addition, prior data on anxiety symptoms from each woman before the pandemic were not available. Although the STAI score makes possible to assess anxiety trait, results may not have adequately measured the real effect of the pandemic and social isolation faced by these women.

\section{CONCLUSIONS}

Periods of uncertainty, stress, and social isolation can increase the risk of depression and anxiety among vulnerable people. This study contributes by expanding the knowledge of the psychological implications of COVID-19 in pregnant women with comorbidities, such as hypertension and diabetes, known risk factors for severe coronavirus infections. Our results demonstrate high rates of anxiety and depression symptoms in this population. Considering that these symptoms can affect both the mother's and offspring's health, we highlight the importance of supporting their mental health, including the identification of risk factors for psychological distress, early recognition of these symptoms, and their treatment. Future studies are needed to rigorously assess the impact of COVID-19 on this vulnerable population in short and long term.

\section{AUTHORS" CONTRIBUTIONS}

RPB: Conceptualization, Project Administration, Writing - Review \& Editing. BDS: Conceptualization, Project Administration, Writing - Review \& Editing. AAJR: Project Administration, Writing - Review \& Editing. GOGM: Project Administration, Writing - Review \& Editing.

\section{REFERENCES}

1. Stein A, Pearson RM, Goodman SH, Rapa E, Rahman A, McCallum $M$, et al. Effects of perinatal mental disorders on the fetus and child. Lancet. 2014;384(9956):1800-19. https:// doi.org/10.1016/S0140-6736(14)61277-0

2. Coelho HF, Murray L, Royal-Lawson M, Cooper PJ. Antenatal anxiety disorder as a predictor of postnatal depression: a longitudinal study. J Affect Disord. 2011;129(1-3):348-53. https://doi.org/10.1016/j.jad.2010.08.002

3. Saccone G, Florio A, Aiello F, Venturella R, Angelis MC, Locci $M$, et al. Psychological impact of coronavirus disease 2019 in pregnant women. Am J Obstet Gynecol. 2020;223(2):293-5. https://doi.org/10.1016/j.ajog.2020.05.003

4. Zheng Z, Peng F, Xu B, Zhao J, Liu H, Peng J, et al. Risk factors of critical \& mortal COVID-19 cases: a systematic literature review and meta-analysis. J Infect. 2020;81(2):e16-25. https:// doi.org/10.1016/j.jinf.2020.04.021
5. Liu H, Wang LL, Zhao SJ, Kwak-Kim J, Mor G, Liao AH. Why are pregnant women susceptible to COVID-19? An immunological viewpoint. J Reprod Immunol. 2020;139:103122. https://doi. org/10.1016/j.jri.2020.103122

6. Grant KA, McMahon C, Austin MP. Maternal anxiety during the transition to parenthood: a prospective study. J Affect Disord. 2008;108(1-2):101-11. https://doi.org/10.1016/j. jad.2007.10.002

7. Manea L, Gilbody S, McMillan D. Optimal cut-off score for diagnosing depression with the Patient Health Questionnaire (PHQ-9): a meta-analysis. CMAJ. 2012;184(3):E191-6. https:// doi.org/10.1503/cmaj.110829

8. Durankuş F, Aksu E. Effects of the COVID-19 pandemic on anxiety and depressive symptoms in pregnant women: a preliminary study. J Matern Fetal Neonatal Med. 2020:1-7. https://doi.org/10.1080/14767058.2020.1763946 
9. Berthelot N, Lemieux R, Garon-Bissonnette J, Drouin-Maziade C, Martel É, Maziade M. Uptrend in distress and psychiatric symptomatology in pregnant women during the coronavirus disease 2019 pandemic. Acta Obstet Gynecol Scand. 2020;99(7):848-55. https://doi.org/10.1111/aogs.13925

10. Yassa M, Yassa A, Yirmibe区 $C$, Birol P, Ünlü UG, Tekin AB, et al. Anxiety levels and obsessive compulsion symptoms of pregnant women during the COVID-19 pandemic. Turk J Obstet Gynecol. 2020;17(3):155-60. https://doi.org/10.4274/ tjod.galenos.2020.91455

11. Sinaci S, Tokalioglu EO, Ocal D, Atalay A, Yilmaz G, Keskin $\mathrm{HL}$, et al. Does having a high-risk pregnancy influence anxiety level during the COVID-19 pandemic? Eur J Obstet Gynecol Reprod Biol. 2020;255:190-6. https://doi.org/10.1016/j. ejogrb.2020.10.055

12. Kim TH, Connolly JA, Tamim H. The effect of social support around pregnancy on postpartum depression among Canadian teen mothers and adult mothers in the maternity experiences survey. BMC Pregnancy Childbirth. 2014;14:162. https://doi. org/10.1186/1471-2393-14-162

13. Davenport MH, Ruchat SM, Poitras VJ, Garcia AJ, Gray CE, Barrowman N, et al. Prenatal exercise for the prevention of gestational diabetes mellitus and hypertensive disorders of pregnancy: a systematic review and meta-analysis. $\mathrm{Br} \mathrm{J}$ Sports Med. 2018;52(21):1367-75. https://doi.org/10.1136/ bjsports-2018-099355

14. Nagpal TS, Mottola MF. Physical activity throughout pregnancy is key to preventing chronic disease. Reproduction. 2020;160(5):R111-8. https://doi.org/10.1530/REP-20-0337

15. Ruchat SM, Mottola MF, Skow RJ, Nagpal TS, Meah VL, James $\mathrm{M}$, et al. Effectiveness of exercise interventions in the prevention of excessive gestational weight gain and postpartum weight retention: a systematic review and meta-analysis. $\mathrm{Br} \mathrm{J}$ Sports Med. 2018;52(21):1347-56. https://doi.org/10.1136/ bjsports-2018-099399

16. Wadhwa Y, Alghadir AH, Iqbal ZA. Effect of antenatal exercises, including yoga, on the course of labor, delivery and pregnancy: a retrospective study. Int J Environ Res Public Health. 2020;17(15):5274. https://doi.org/10.3390/ijerph17155274
17. Biviá-Roig G, La Rosa VL, Gómez-Tébar M, Serrano-Raya L, Amer-Cuenca JJ, Caruso S, et al. Analysis of the impact of the confinement resulting from COVID-19 on the lifestyle and psychological wellbeing of spanish pregnant women: an internet-based cross-sectional survey. Int J Environ Res Public Health. 2020;17(16):5933. https://doi.org/10.3390/ ijerph17165933

18. Rezende LFM, Sá TH, Mielke GI, Viscondi JYK, Rey-López JP, Garcia LMT. All-cause mortality attributable to sitting time: analysis of 54 countries worldwide. Am J Prev Med. 2016;51(2):253-63. https://doi.org/10.1016/j.amepre.2016.01.02219

19. Fazzi C, Saunders DH, Linton K, Norman JE, Reynolds RM. Sedentary behaviours during pregnancy: a systematic review. Int J Behav Nutr Phys Act. 2017;14(1):32. https://doi.org/10.1186/ s12966-017-0485-z

20. The Lancet. COVID-19 in Brazil: "So what?". Lancet. 2020;395(10235):1461. https://doi.org/10.1016/S01406736(20)31095-3

21. New England Journal of Medicine Editors. Dying in a leadership vacuum. N Engl J Med. 2020;383(15):1479-80. https://doi. org/10.1056/NEJMe2029812

22. Instituto Brasileiro de Geografia e Estatística. Diretoria de pesquisas, coordenação de trabalho e rendimento, pesquisa nacional por amostra de domicílios contínua (2017-2018). Rio de Janeiro: IBGE; 2018. [cited on Oct. 20, 2020]. Available from: https://www.ibge.gov.br/estatisticas/sociais/populacao/17270pnadcontinua.html? edicao $=27138$

23. International Telecommunication Union. Measuring digital development facts and figures 2019. Geneva: International Telecommunication Union; 2019. [cited on Oct. 20, 2020]. Available from: https://www.itu.int/en/ITU-D/Statistics/ Documents/facts/FactsFigures2019.pdf

24. Fine TH, Contractor AA, Tamburrino M, Elhai JD, Prescott MR, Cohen $\mathrm{GH}$, et al. Validation of the telephone-administered PHQ-9 against the in-person administered SCID-I major depression module. J Affect Disord. 2013;150(3):1001-7. https://doi.org/10.1016/j.jad.2013.05.029

25. Carr ECJ, Worth $A$. The use of the telephone interview for research. J Res Nurs. 2001;6(1):511-24. https://doi. org/10.1177/136140960100600107 